\title{
Impact of Climate Change on Schizophrenia Patients with Sex Stratifications: Variability of Temperature and Relative Humidity Effects
}

\author{
Olatunji Paul Jaiyeola ${ }^{1}$, Abdullahi Ayegba ${ }^{2}$, Funmilola A. Oluwafemi ${ }^{3}$, Irene Benibo ${ }^{4}$ \\ ${ }^{1,4}$ National Centre for Remote Sensing (NCRS), Jos, Nigeria \\ ${ }^{2,3}$ Engineering and Space System (ESS) Department (NASRDA), Abuja, Nigeria \\ ${ }^{1,2,3,4}$ National Space Research and Development Agency (NASRDA), Abuja, Nigeria \\ Department of Biochemistry, Federal University Oye Ekiti (FUOYE), Oye-Ekiti State, Nigeria \\ DOI: 10.29322/IJSRP.10.11.2020.p10763 \\ http://dx.doi.org/10.29322/IJSRP.10.11.2020.p10763
}

\begin{abstract}
The impact of temperature and relative humidity on sex differences among schizophrenia patients at Neuropsychiatric Hospital, Akure, and Ondo State, Nigeria was examined in this paper. Two independent variables (temperature and relative humidity) and one dependent variable (number of schizophrenia patients that visited the hospital from (2010-2017) data were collected. The population of study were 13,834 (in-patients and out-patients) for the period of eight years; that comprised 7,290 $(52.70 \%)$ female and 6,544 (47.30\%) male schizophrenia patients. The collected data were analyzed with the use of single regression and multivariate regression analyses. This study therefore, found that climate parameters: relative humidity and temperature had overlapping effects on the number of male and female schizophrenia patients that consulted at the hospital. It was found that increase in temperature had negative association with the number of schizophrenia patients i.e., both sexes were vulnerable to the risk of schizophrenia (mental ill-health); and that the number of female schizophrenia patients recorded for the eight years was higher in number compared to the male schizophrenia patients. It was also observed that the vulnerability of males to the risk of schizophrenia with reference to relative humidity increased and differed from the female vulnerability. This is an indication that other factors - bio psychosocial, environmental, culture etc. may not have made male and female population equally vulnerable at the risks of schizophrenia. The probability that the male population is more vulnerable to the risk of schizophrenia cases as relative humidity and overlapping effect increases is 0.13 and 0.22 respectively for male while that of female is 0.12 and 0.15 respectively. The number of schizophrenia patients diagnosed and traced to increase in relative humidity and overlapping effect was 2,041 females and 3,099 males; 2,019 females and 1,718 males respectively. This might be due to the fact that: male patients respond to treatment very slowly as compared to females; the recovery period of males with schizophrenia was longer than for females; consequently, the rate of discharge from hospital for male schizophrenia patients was lower than the discharge of the female schizophrenia patients; the cognitive deficits and memory delay was more in male schizophrenia patients than the female. Therefore, all these can aggravate as relative humidity increases coupled with the: risk of psychoactive substance use; the
\end{abstract}

protective effect of estrogen is lower in male than female; psychoactive substance consumption rate is higher in male than in female. Also, increasing rate of schizophrenia is caused as relative humidity increases because testosterone reduction in male occurs during hot weather, and reduction in the brain gray matter in male tend to reduce neuron density.

Index Terms- Temperature, Relative Humidity, Overlapping Effect and Sex Distribution of Schizophrenia Patients

\section{INTRODUCTION}

C chizophrenia was introduced at the beginning of this century as a term into the medical language by the Swiss psychiatrist Bleuler. Schizophrenia is a major mental disorder, or a group of disorders with unknown causes that involves a complex set of disturbances of perception, thinking and social behavior. It has been discovered that no culture or society in the world is free from schizophrenia, and evidence states that this illness is a serious public health problem (Barbato, 1998). Also, according to Aleman (2014) schizophrenia belongs to the most severe psychiatric disorders and it is characterized by hallucination, delusions, cognitive impairment, blunted emotion, disordered thinking, and social withdrawal accompanied by intellectual disturbances and other emotional behaviors. Aleman explained further that patients with schizophrenia suffer from significant deficit in attention, memory, executive functioning, and general intellectual abilities and these can be manifested in such manners as having difficulty to: think clearly; distinguish reality from fantasy; react in an emotionally inappropriate way; and to interact with others. Schizophrenia is often associated with dopamine imbalances in the brain (Encyclopaedia Britannica, 2020). Characteristics of schizophrenia are of two different types:

Type 1- Positive Symptoms: This is characterized with things such as delusions, hallucinations and paranoia. Patients may experience things such as hearing voices, which are often said to be from God or devils.

Type 2- Negative Symptoms: This is characterized with or shows more of catatonic behavior. Patients exhibit a loss of 
drive, lack of emotion or catatonic stupor. Catatonic simply mean not able to move or show any reaction to things because of mental illness especially schizophrenia, in which a person does not move for long periods.

Hafner (2002) compared clinical subtypes, analyzed symptomatology, type of illness onset and course from the first sign to the climax of the first episode. The three types of onset: acute, subacute, and chronic; and three categories of initial symptoms: positive, negative and unspecific. Although, patients can be diagnosed with both types 1 and 2 symptoms. When this happens, it is been categorized as disorganized as they will exhibit disorganization, even in speech.

Recent studies have shown that environmental factors might be involved in the development of schizophrenia. The importance of these factors has been demonstrated by the fact that in monozygotic twins discordant for schizophrenia, environmental variations might have resulted in inter-individual differences and that current etiological models regarding the pathogenesis of this disease propose interactive effects between multiple genes and environmental factors (Fernanda et al., 2009). Although various theories of the origin of schizophrenia have centered on anatomical, biochemical, psychological, social, genetic and environmental causes, but evidence strongly shows that a combination of genetic and environmental factors play an important role in the development of the condition. There is a $1 \%$ chance of developing schizophrenia if neither of the parents carries the gene, however this increases to $20 \%$ if one of the parents had positive history of schizophrenia, and increases to $46 \%$ if both parents had exhibited positive acts of schizophrenia.(any authority)?

According to Shiloh et al. (2005a) the potential effects of climate on the severity of schizophrenia symptoms are not well established while some data suggest higher summer admissions of schizophrenia patients to psychiatric facilities and other data state that there are various factors (e.g. stress of vacation, family being away, staff away, least support in community and health services) that increased patients admission in hospitals. Specific climatic factors (ambient temperature, relative humidity and photoperiod) are assumed to contribute to the emergence of psychotic exacerbation and increased summer admissions. Most of these studies grouped together schizophrenic and affective psychoses, thus excluding an analysis of their potentially distinct admission patterns. Hence in this study, it is hypothesized that various climatic factors may contribute to the emergence of psychosis in schizophrenia patients and consequently may increase their admission rates to psychiatric hospitals.

Schizophrenia was among disorders that were associated with increased frequency of heat-related-illness (HRI) hospitalization among mental illness and behavioral disorder (MBD) patients. Increased risk of mental behavioral disorder (MBD) was observed in males. Previous studies outside U.S have found that dementia and schizophrenia are significant factors for heat-related-illnesses (Schmeltz and Gamble, 2017). Shiloh et al., (2005b) found that mean monthly admission rates were significantly higher during the summer for schizophrenia patients and the fall season for schizo-affective patients. Schizophrenia patients' mean maximal monthly environmental temperature correlated with mean monthly admission rates. This equally indicates that persistent high environmental temperature may be a contributing factor for psychotic exacerbation in schizophrenia patients and their consequent admission to mental hospitals.

Extreme warming affects schizophrenia, but cooling does not (Aleman et al., 2016) and extremely high diurnal temperature range (DTR) is a potential trigger for schizophrenia admission in Hefei, China (Zhao et al., 2016). According to Lee et al. (2017), mental illness is important to consider among risk factors for heat-related morbidity with increased hospital admissions during extreme heat events and that there are numerous studies from Australia, Canada, Israel and other countries examining the association between high temperature and hospitalization related to mental-behavioral-disorder indicating an increased risk temperature related death and hospitalization especially among those with dementia and schizophrenia. Several studies have identified more cases of patients with schizophrenia in the summer. Research has shown a seasonal influence on first admission for schizophrenia (in the Northern Hemisphere including Ireland, England and Scotland) and the peak time of the hospital entries for mental disorders occurred more about the middle of the year (Amr et al., 2012; Shiloh et al., 2009). There was significant relationship between higher ward temperature and more severe symptoms among in-patients with schizophrenia from the research conducted in Israel (Shiloh et al., 2009).

When considering the schizophrenia symptoms and onset of the illness, Hafner (2002) stated that most studies reported a greater frequency of positive and affective symptoms for women and more negative symptoms and insidious types of onset for men. According to Li et al. (2017), men with schizophrenia appear to have more negative symptoms and more severe clinical features than females, particularly in social withdrawal, substance abuse and blunted or incongruent effects. Women with schizophrenia often have more mood disturbances, depressive symptoms and affective symptoms. In the case of sex differences in the onset of the illness, Ochoa et al., (2012) wrote, men usually develop the illness at age 18-25, while in women, the mean age of onset is 25-35 and that women seem to have two peaks in the age of onset of the diseases; the first after menarche and the second when they are over 40. According to Li et al. (2017), men have single peak age for onset which is between 21 25 years old and women have two peaks age of onset, one between 25 and 30 years old and another is after 45 years old. Aleman (2003) acknowledged that in younger age groups, the risk is higher for men and that beyond age 40 years and perhaps even earlier, the risk is higher for women, but according to Hafner (2002), there are steep increase with a maximum between 15 and 25 years for men and after that a monotonous decrease to a very low level. While in women the rate of onsets rose slightly less steeply and reached a lower and broader peak in age band 15 to 30. After a decline, female onset reached a second somewhat smaller peak in age group 45 to 50 around pre-menopause with significant difference to men. The three researchers and their group Ochoa, Li, Hafner and Aleman showed that men have single peak and women have two peaks. The peaks differences can be suggested to be in the physiological and psychological operational differences in relating to body chemical and biological functions, and their interaction with environmental and social elements. 
According to Hafner (2002), normal early childhood development differs very little between the sexes. In the late childhood, boys exhibit more externalizing behaviors and a slightly higher frequency of attention deficits, and girls have more anxiety from puberty. Therefore, the mental health risks of males and females follow different lines, males show a greater frequency of hyperactivity, attention deficit disorder, dissocial behavior, aggressiveness and anti-social personality disorder, while females show greater frequency of anxiety and affective disorders. Externalizing disorders including antisocial behavior and substance abuse are more frequent in men, while internalizing disorder including anxiety and depression are more frequent in women.

Adult-onset schizophrenia is preceded by mild neuromotor, cognitive and behavior anomalies. In the minors, early childhood deficits in neuromotor and speech development as antecedents of schizophrenia seem to occur at the same frequency in boys and girls, but the behavioral anomalies manifest themselves several years later in girls than in boys most especially from the school age. The severities of these anomalies are more in children of mothers with schizophrenia and boys clearly score higher than girls on cognitive impairment.

\section{Methodology}

Secondary data were drawn from two sources and used for this study, at Neuropsychiatric Hospital, Akure, Ondo State,
Nigeria where the monthly and the yearly number of in-patients and out-patients admitted with schizophrenia or mental ill health from the year 2010 to 2017 were collected, and meteorological data on temperature and relative humidity were used. The meteorological data on temperature and relative humidity of Ondo State from 2010 to 2017 were retrieved from the ModernEra retrospective analysis for Research Application, Version 2 (MERRA-2) web site. The ex-post facto research design was adopted for the study, and the data collected were analyzed with the use of STATA: Single and Multivariate regression analysis. Total number of in-patients and out-patients visiting the hospital with schizophrenia for eight years (2010-2017) was 13834, comprising $6544(47.30 \%)$ male and $7290(52.70 \%)$ female schizophrenia patients. Internet facility was employed for this research, especially e-library to retrieve text books, journals, research papers etc. for an update information on the topic from other researchers and related studies e.g. Google Alerts and Google Trends to rack comprehensive search over lengthy period.

\section{DATA PRESENTATION AND ANALYSIS}

\subsection{Data Presentation}

Table 1: Numbers of Male and Female Schizophrenia Patients, temperature and Humidity

\begin{tabular}{|c|c|c|c|c|}
\hline & & Sex & Climate Para & \\
\hline & $\begin{array}{l}\text { 2010-2017 } \\
\text { Numbers of } \\
\text { male } \\
\text { Schizophrenia } \\
\text { Patients }\end{array}$ & $\begin{array}{l}\text { 2010-2017 Numbers } \\
\text { of Female } \\
\text { Schizophrenia } \\
\text { Patients }\end{array}$ & Temperature & Humidity \\
\hline Jan & 460 & 508 & 24.50 & 76.75 \\
\hline Feb & 510 & 518 & 25.88 & 82.87 \\
\hline March & 424 & 586 & 26.25 & 86.30 \\
\hline April & 489 & 561 & 26.13 & 88.13 \\
\hline May & 661 & 713 & 25.71 & 89.80 \\
\hline June & 611 & 596 & 24.96 & 90.02 \\
\hline July & 483 & 693 & 24.32 & 89.36 \\
\hline August & 733 & 627 & 24.11 & 88.70 \\
\hline September & 591 & 546 & 24.50 & 89.53 \\
\hline October & 603 & 713 & 25.13 & 89.24 \\
\hline
\end{tabular}

This publication is licensed under Creative Commons Attribution CC BY. 


\begin{tabular}{|l|l|l|l|l|} 
& & & & \\
\hline November & 507 & 606 & $\mathbf{2 5 . 5 3}$ & $\mathbf{8 6 . 4 5}$ \\
\hline December & 472 & 623 & $\mathbf{2 4 . 3 9}$ & $\mathbf{7 7 . 3 8}$ \\
\hline Total & 6544 & 7290 & & \\
\hline
\end{tabular}

\subsection{Data Analysis}

\subsubsection{Single Regression Analysis}

Table 2: Data from STATA Regression Analysis

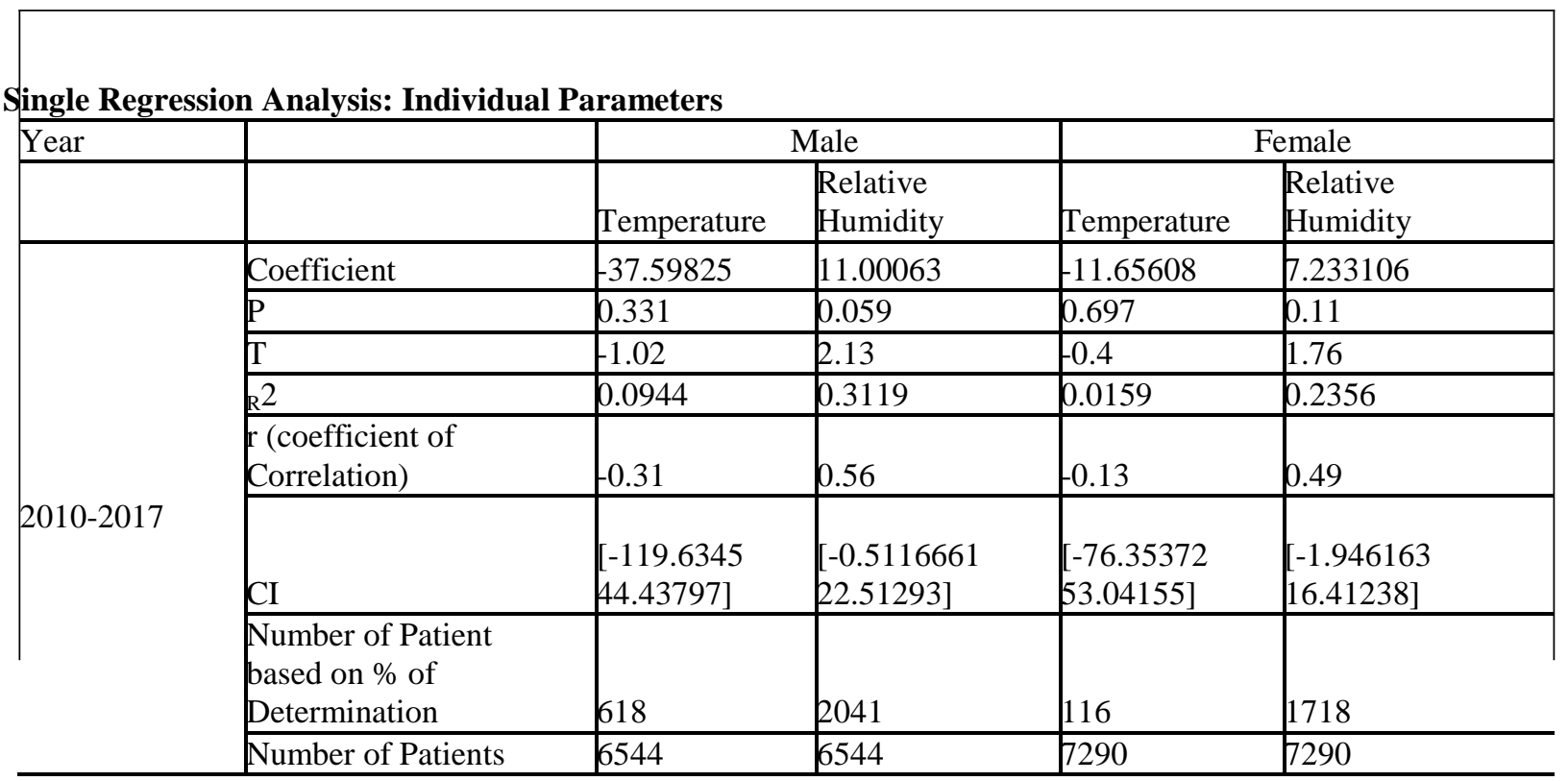

\section{From table 2 above:}

\section{Male Schizophrenia Patients}

Temperature as a parameter in this study had a negative impact. The coefficient for temperature shows that an increase in temperature resulted to 37.60 decrease in number of male schizophrenia patients but was statistically not significant with confidence interval [-119.635 44.438] i.e. the reduction in the number of male schizophrenia patients may be as much as 120 or there could be an increase in number of male schizophrenia patients as low as 44 as the temperature increased. This decrease in the number of male schizophrenia patients due to increase in temperature is not statistically significant because $\mathrm{p}(0.331)$

$>\quad 0.05$ and the $t(1.02)<2.04$. The amount of reduction in number of male schizophrenia patients is $9 \%$ (i.e. $\mathrm{R}^{2}$ ). Increase in the temperature reduced the number of male schizophrenia patients with $9 \%$ of 6544 which equals 618 . The negative coefficient of correlation $r(0.31)$ is weak.

The relative humidity parameter comparatively had a positive effect. The coefficient for relative humidity shows that an increase in relative humidity resulted to 11 decrease in number of male schizophrenia patients but statistically not significant with confidence interval $\left[\begin{array}{lll}-0.5117 & 22.5129\end{array}\right]$ i.e. the reduction in the number of male schizophrenia patients may be as less as 1 or there could be an increase in number of male schizophrenia patients was raised to 23 when relative humidity increased. This increase in the number of male schizophrenia patients due to increase in the relative humidity is not statistically significant because $p(0.06)>0.05$. The increase in the number of male schizophrenia patients was $31.19 \%$ (i.e. $\mathrm{R}^{2}$ ). Increase in relative humidity increased the number of male schizophrenia patients by

$31.19 \%$ of 6,544 which equals 2,041. The positive coefficient of correlation $r(0.56)$ between increase in the relative humidity and the number of male schizophrenia patients is moderate.

\section{Female Schizophrenia Patients}

The temperature parameter also had a negative effect on the female patients. The coefficient for temperature shows that an increase in temperature resulted in 11.656 decrease in the number of female schizophrenia patients but statistically not significant 
with confidence interval [-76.354 53.042] i.e. the reduction in the number of female schizophrenia patients may be as much as 76 or there could be an increase in number of female schizophrenia patients as low as 53 as temperature increased. This decrease in the number of female schizophrenia patients due to increase in the temperature was not statistically significant because $\mathrm{p}(0.697)$ $>0.05$ and the $\mathrm{t}(-0.4)<2.04$. The amount of reduction in number of female schizophrenia patients is $1 \%$ (i.e. $\mathrm{R}^{2}$ ). Increase in the temperature reduced the number of female schizophrenia patients with $1 \%$ of 7290 which equals 116. The negative coefficient of correlation $\mathrm{r}(0.31)$ is very weak.

The relative humidity parameter impacted positively. The coefficient for relative humidity showed that an increase in relative humidity resulted in 7.233 decrease in number of female schizophrenia patients but statistically not significant with confidence interval $[-1.94616 .412]$ i.e. the reduction in the number of female schizophrenia patients may be as less as 2 or there could be an increase in number of female schizophrenia patients as high as 16 as relative humidity increases. This increase in the number of female schizophrenia patients due to increase in the relative humidity is not statistically significant because $\mathrm{p}(0.11)>0.05$ and $\mathrm{t}(1.76)<2.04$. Increase in the number of female schizophrenia patients was $23.56 \%$ (i.e. $\mathrm{R}^{2}$ ). Increase in the relative humidity increased the number of female schizophrenia patients with $23.56 \%$ of 7,290 which equals 1,718 . The positive coefficient of correlation $r(0.49)$ between increase in the relative humidity and the number of female schizophrenia patients was moderate.

\subsubsection{Multivariate Regression Analysis:}

Table 3: Data from STATA Regression Analysis

\section{Multivariate Regression Analysis: Individual Parameters}

\begin{tabular}{|c|c|c|c|c|c|}
\hline \multirow[t]{2}{*}{ Year } & & \multicolumn{2}{|c|}{ Male } & \multicolumn{2}{|c|}{ Female } \\
\hline & & Temperature & $\begin{array}{l}\text { Relative } \\
\text { Humidity }\end{array}$ & Temperature & $\begin{array}{l}\text { Relative } \\
\text { Humidity }\end{array}$ \\
\hline \multirow{10}{*}{2010} & Coefficien & -49.84673 & 12.28834 & -19.36435 & 7.233106 \\
\hline & $\mathrm{P}>(\mathrm{t})$ & 0.131 & 0.031 & 0.484 & 0.11 \\
\hline & $T$ & -1.66 & 2.55 & -0.73 & 1.76 \\
\hline & $\mathrm{F}$ & 4.049052 & 4.049052 & 1.735007 & 1.735007 \\
\hline & $\mathrm{P}$ & 0.0557 & 0.0557 & 0.2305 & 0.2305 \\
\hline & $\mathrm{R}^{2}$ & 0.4736 & 0.4736 & 0.2783 & 0.2783 \\
\hline & $\begin{array}{l}\text { Constant } \\
\text { (intercept) }\end{array}$ & \multicolumn{2}{|c|}{$\begin{array}{l}t: 0.92, P>(t): 0.380, C I: 737.9708[- \\
1071.7172547 .659]\end{array}$} & \multicolumn{2}{|c|}{$\begin{array}{l}t: 0.60, P>(t): 0.562, C I: 427.1854[- \\
1175.9062030 .277]\end{array}$} \\
\hline & CI & {$\left[\begin{array}{l}-117.6603 \\
17.96688]\end{array}\right.$} & $\begin{array}{l}{[1.371108} \\
23.20556]\end{array}$ & $\begin{array}{l}{[79.43626} \\
40.70756]\end{array}$ & {$\left[\begin{array}{l}-1.93775 \\
17.40425]\end{array}\right.$} \\
\hline & $\begin{array}{l}\% \text { of } \\
\text { Determina } \\
\text { tion }\end{array}$ & $\beta 099$ & 3099 & 2029 & 2029 \\
\hline & $\begin{array}{l}\text { Number of } \\
\text { Patients }\end{array}$ & 6544 & 6544 & 7290 & 7290 \\
\hline
\end{tabular}

\section{From Table 3 above:}

\section{Male Schizophrenia Patients}

In the overlapping process, the coefficient for temperature showed that an increase in temperature resulted in 49.847 decrease in the number of male schizophrenia patients but it was statistically not significant with confidence interval [-117.550 17.967] i.e. the reduction in the male schizophrenia patients may be as much as 118 or there could be an increase in the number of male schizophrenia patients as low as 18 .
In the overlapping process, the coefficient for relative humidity showed that an increase in relative humidity led to an increase of 12.288 in the number male schizophrenia patients, and was statistically significant at a confidence interval of [1.37123 .206 ] i.e. the reduction in the number of male schizophrenia patients could be as low as 1 or there could be an increase in the number of male schizophrenia patients as much as 23. 
The effect of the two independent variables on the number of the male schizophrenia patients were not the same i.e. increase in the temperature has negative impact on the number of male schizophrenia patients, because the magnitude of the decrease in the number of male schizophrenia patients due to the increase in temperature was more than the magnitude of the increase in the number of male schizophrenia patients and increase in the relative humidity has positive impact on the number of the male schizophrenia patients, because the magnitude of the increase in the number of the male schizophrenia patients due to the increase in relative humidity is more than the magnitude of the decrease in the number of male schizophrenia patients.

In the overlapping processes, the increase in the relative humidity increases the number of male schizophrenia patients positively than the increase in temperature

The multivariate regression analysis yielded overlapping effect with the value of intercept at vertical axis of positive confidence interval 737.971[-1071.717 2547.659]. The intercept on the number of patients axis (vertical axis) for the two parameters shows that an increase in the two parameters gave as much as 738 increase in the number of male schizophrenia patients, and a decrease in the number of male schizophrenia patients was as low as 1,072 or there could be an increase in the number of male schizophrenia patients as much as 2,547 but was statistically not significant with $\mathrm{p}(0.380)>0.05$ and $\mathrm{t}(0.92)<$ 2.04. The overall impact of the overlapping effect of the increase in the two parameters impacted positively i.e. the magnitude of increase in the number of male schizophrenia patients due to increase in the two parameters was more than the magnitude of decrease in the number of male schizophrenia patients. Also, F $(4.049)>t$ (2.04) and F carried positive value. The overlapping effect of the increase in the two independent variables therefore, increased the number of male schizophrenia patients. However, there was an increase in the overlapping effect of the two parameters increase the number of male schizophrenia patients by $47.36 \%$ (from $\mathrm{R}^{2}$ ). The increase in the number of male schizophrenia patients due to overlapping effect was $47.36 \%$ of 6544 which equaled 3099.

\section{Female Schizophrenia Patients}

In the overlapping process, the coefficient for temperature showed that $\mathrm{x}$ increase in temperature resulted in 19.364 decrease in the number of female schizophrenia patients, but was statistically not significant with confidence interval [-79.436 40.708] i.e. the reduction in the female schizophrenia patients may be as much as 79 or there could be an increase in the number of female schizophrenia patients as low as 41 .

In the overlapping process, the coefficient for relative humidity showed that an increase in relative humidity will resulted to 7.233 increase in the number female schizophrenia patients, but was statistically significant with confidence interval [-1.938 17.404] i.e. the reduction in the number of female schizophrenia patients was as low as 2 or there could be an increase in the number of the female schizophrenia patients as much as 17 .

The effect of the two independent variables on the number of the female schizophrenia patients were not the same i.e. increase in the temperature has negative impact on the number of the female schizophrenia patients, because the magnitude of the decrease in the number of the female schizophrenia patients due to increase in temperature is more than the magnitude of increase in the number of the female schizophrenia patients; and the increase in the relative humidity had positive impact on the number of the female schizophrenia patients, because the magnitude of the increase in the number of the female schizophrenia patients due to the increase in relative humidity was more than the magnitude of the decrease in the number of the female schizophrenia patients.

In the overlapping processes, the increase in the relative humidity increases the number of female schizophrenia patients positively than the increase in temperature

The multivariate regression analysis yielded overlapping effect with the value of intercept at vertical axis with positive confidence interval 427.1854[-1175.906 2030.277]. The intercept on the number of patients' axis (vertical axis) for the two parameters shows that an increase in the two parameters will lead to 427 increase in the number of female schizophrenia patients and that the decrease in the number of female schizophrenia patients could be as less as 1180 or there could be an increase in the number of female schizophrenia patients as much as 2030; but statistically not significant with $\mathrm{t}: 0.60, \mathrm{P}>(\mathrm{t}): 0.562$ or $\mathrm{P}$ $(0.562)>0.05$ and $t(0.60)<2.04$. The overall impact of the overlapping effect of the increase in the two parameters is of positive impact i.e. the magnitude of increase in the number of female schizophrenia patients due to the increase in the two parameters is more than the magnitude of decrease in the number of female schizophrenia patients. Also, F (1.735) > t (2.04) and F carried positive value. The overlapping effect of the increase in the two independent variables increases the number of female schizophrenia patients. The increase in the overlapping effect of the two parameters increased the number of male schizophrenia patients by $27.83 \%$ (from $\mathrm{R}^{2}$ ). The increase in the number of the male schizophrenia patients due to overlapping effect is $27.83 \%$ of 7290 which equals 2,029 .

\section{FINDINGS}

In table 2 above, although temperature contributed nothing to increase the number of schizophrenia patients in both sexes, the rate at which the number of male schizophrenia patients decreased as the temperature increased was higher than the rate at which the number of female schizophrenia patients decreased. Notwithstanding the number of male and female schizophrenia patients have negative association with increase in temperature. In other words, none of 6544 and 7290 total numbers of male and female schizophrenia patients respectively attributed to the increase in temperature. None of the diagnoses were traceable to increase in temperature. Male and female schizophrenia patients visiting the Neuropsychiatric Hospital, Akure, Nigeria between 2010 and 2017 did not result from increase in temperature.

It can be deduced therefore that increase in relative humidity increased the number of schizophrenia patients visiting the hospital in both sexes. The number of male and female schizophrenia patients had strong correlation (in terms of their correlation coefficients) with increase in relative humidity, but 
the rate at which schizophrenia psychiatric cases per unit relative humidity spread among males was more than the females. Even, $\mathrm{t}$ (2.13) for males was greater than $t$ (1.76) for females. Increase in the relative humidity made male population more vulnerable to the risk of schizophrenia psychiatric cases than the female population. There were four parameters that really showed that the male schizophrenia patients were more than the female patients as the relative humidity increased. The four parameters are:

- $\quad$ The $\mathrm{t}$-value where $\mathrm{t}($ male $)>\mathrm{t}$ female).

- The correlation coefficients $r$ : where $r(0.56)$-male $>r$ (0.49)-female.

- The Confidence Interval, CI: was checked and the width ( ) of the lower and upper interval or the distance from the point of the upper interval from the lower interval in magnitude was only CL: (23.025)-male > (18.359)-female.

- The number of patients based on the $\%$ of determination male (2041) > Female (1718).

From Table 3 above, the multivariate regression analysis gives the overlapping effect of the two independent variables

In the overlapping process, the major factor that contributed to the elevated number of male and female schizophrenia patients in hospital was increase in relative humidity because the number of male and female schizophrenia patients had positive coefficient i.e. male and female schizophrenia patients have positive association with relative humidity as the parameters are in overlapping process. The effective strength of the increase in the relative humidity was more the males than the females in the overlapping processes and this is established from $\mathrm{t}$ (2.55)-male $>\mathrm{t}$ (1.81)-female. The overall event of the overlapping effect of the two independent variables on the number of male and female schizophrenia patients showed positive association with the number of schizophrenia patients visiting the hospital, and this is also established from the F-value that shows positive sign. The strength effect of the overlapping of the two-independent variable of interest is more on the male than the female and is established from:

- $\quad$ F (4.05)-male > F (1.74)-female.

- At the intercept: $\mathrm{t}(0.92)$-male $>\mathrm{t}(0.60)$-female.

- At the intercept-Y-axis: Number of schizophrenia patients for male (738) and female (427).

- At the intercept-Y-axis, CI: The number of male schizophrenia patients can be reduced to as less as 1,072 and can be increased to as much as 2548 while the number of female schizophrenia patients can be reduced to as less as 1176 and can be increased to as much as 2030 .

The reduction in the number of the female schizophrenia patients is more than the reduction in the male schizophrenia patients.

This publication is licensed under Creative Commons Attribution CC BY.

http://dx.doi.org/10.29322/IJSRP.10.11.2020.p10763
The increase in the male schizophrenia patients is more than the female schizophrenia patients.

Combining the two above shows that the number of the male schizophrenia patients will be more than the number of the female schizophrenia patients.

- $\%$ of Determination: The number (3099) of the male schizophrenia patients is more than the number (2029) of the female schizophrenia patients as the overlapping effect increases.

\section{DISCUSSION OF FINDINGS}

The number of schizophrenia patients visiting the hospital from 2010-2017 were more of female (7290) than male (6544), while the total is 13834 . The probability that the schizophrenia patients were male is 0.47 and the probability that the schizophrenia patients were female is 0.53 . When all possible factors that can cause schizophrenia mental illness are considered, the female populations are discovered to be more vulnerable to the risk of schizophrenia cases than male. It can be that the relative risk or incidence risk of female population is equivalent to 1.13 compared with the male population visiting Neuropsychiatric Hospital, Akure, Nigeria in respect of the factors behind the schizophrenia cases.

The sex differences have been attributed to biological, psychosocial, and cultural factors (Novick et al., 2016) called biopsychosocial causes of the sex differences (Falkenburg and Tracy, 2012). It is also believed that the biopsychosocial and cultural differences can make each sex (male and female) respond

differently to environmental factors e.g. climate change (temperature and relative humidity). The number of patients visiting the hospital from table 1 was recorded based on the combination of many factors and the records shows that female schizophrenia patients were more than male patients, but with specific factors such as climate change (temperature and relative humidity as the two parameters of interest), it was discovered that male schizophrenia patients diagnosed were traced to relative humidity and were more than the female patients. While the increase in temperature made no contribution to the number of schizophrenia patients (in either male or female) from the regression analysis; the overlapping effect does make both sexes vulnerable to the risk of schizophrenia cases. From the regression analysis and overlapping effects of the two independent variables, it is seen that different factors can make male or female schizophrenia patients more vulnerable at the risk of schizophrenia cases than the other.

The total schizophrenia cases diagnosed and traced to climate change was 3,759 from single regression analysis; it comprised 2,041-male and 1,718-female while the total number of schizophrenia patients visiting the hospital for a period of eight years (2010 to 2017) was 13834. None of the male or female schizophrenia patients were traced to increase in temperature. The contribution of increased temperature as a separate entity was zero.

- The probability that temperature (T) makes male (M) population vulnerable at the risk of schizophrenia mental illness out of all schizophrenia patients visiting hospital for eight 
years was ()$=0$; and the probability that relative humidity $(\mathrm{RH})$ makes male population vulnerable at the risk of schizophrenia mental cases is ()$={ }_{13834}^{2041}=0.13$. The probability that temperature or relative humidity makes male population vulnerable to schizophrenia cases is ()$=()+()=0.13$ mainly from the diagnosis that was traced to increase in the relative humidity.

- $\quad$ The probability that temperature $(\mathrm{T})$ makes female $(\mathrm{F})$ population vulnerable at the risk of schizophrenia mental illness out of all schizophrenia patients visiting the hospital for eight years is ()$=0$; and the probability that relative humidity $(\mathrm{RH})$ makes female population vulnerable at the risk of schizophrenia mental cases is ()$={ }_{13834}{ }^{1718}=0.12$. The probability that temperature or relative humidity makes male population vulnerable to schizophrenia cases is ()$=()+()=0.12$ mainly from the diagnosis that was traced to increase in the relative humidity.

The probability that the male populations are vulnerable to the risk of schizophrenia cases is higher than the female probability as climate changes is an indication that male populations diagnosed and traced to climate change are more. The effective strength of relative humidity is more on male than female in schizophrenia cases.

(M) This is evident in the overlapping effect. The probability that overlapping effect $(\mathrm{OE})$ makes male population vulnerable to the risk of schizophrenia mental illness out of all schizophrenia patients visiting hospital for eight years is ( ) = $13834^{3099}=0.22$ and the probability that overlapping effect $(\mathrm{OE})$ makes female (F) population vulnerable at the risk of schizophrenia mental illness out of all schizophrenia patients visiting hospital for eight years is $(\quad)=$ $13834^{2029}=0.15$

The followings can be considered to be the reasons why males in the population were more vulnerable to schizophrenia than female population as relative humidity increases are:

- According to Novick et al. (2016), women tend to have higher rates of response, remission, and recovery; lower rates of hospitalization, shorter lengths of stay, longer time of relapse and better social adjustment than men. It can be agreed upon that the rate at which women have been discharged in the hospital is more than men. The population of male are more at a particular time than female as relative humidity increases, since women responded to treatment than men. Women patients with schizophrenia mental cases reduced rapidly than men. Women with schizophrenia therefore have a more favorable course and outcome than men.

- Male schizophrenia patients had more serious cognitive deficits than females in immediate and delay memory (Li et al., 2017), and increased relative humidity and heat stress are observed to significantly impair complex cognitive tasks such as working memo that even worsen insomnia (Cooper, 2018).
- Lack of estrogen protective effect in males can be aggravated with increase in relative humidity, and this can make males to develop the most severe form of schizophrenia disorder early than female. Estrogen plays possible neuroprotective roles against schizophrenia pathology in women. In the microgravity and higher radiation environment where there is possibility of increased relative humidity, estrogen activities can be on decrease, this estrogen deficiency has been found to be highly related to severity of psychiatric symptoms in women during menopause and that during menstrual cycle female schizophrenia patients often have severe symptoms in the low estrogen phase of their menstrual cycle (Li et al., 2017).

- Since the consumption of substance abuse is on the increase as relative humidity and temperature increases, substance abuse presents a higher prevalence in people with schizophrenia and first-episode psychosis; and as the rate at which male population is vulnerable at the risk of schizophrenia cases increase as relative humidity increases. This is an evidence that males consume more psychoactive substance than females; and that men are more vulnerable to the risk of psychoactive substance than female as relative humidity increases (Jaiyeola et al., 2020). Men present higher levels of cannabis, cocaine, hallucinogen consumption and case of alcohol abuse more than women (Hafner, 2002). The percentage of current alcohol or substance abuse was also generally lower in females than in males across all region (Novick et al., 2016).

- One of the symptoms of schizophrenia is frequent hyperactivity and higher frequency of aggressive behaviors, in particular antisocial aggression which is more in display in men than women (Hafner, 2002), most especially men with psychoactive substance use as temperature and relative humidity increases.

- Sex differences in hormone, chromosome, brain structure and activities might bring difference in response to ambient relative humidity and temperature resulting into difference in schizophrenia symptom effect such as negative and positive symptoms. Reduction in the testis weight and testosterone that was found in human during flight and post flight. Without gravity the testes would sit closer to the body and without any air convection they would be at a higher temperature, which could impair sperm production in mammals. In other words, increase in temperature and relative humidity can decrease testosterone; and it was discovered that schizophrenia patients with low levels of testosterone often predominantly have negative symptoms and serum testosterone levels associated with greater severity of negative symptoms of schizophrenia (Li et al., 2017 ) in men. Reduction in oxytocin levels that is responsible for production functions can be found to develop negative schizophrenia symptoms while schizophrenia with higher levels of plasma oxytocin develop fewer psychotic symptoms. This oxytocin can be impaired as temperature and relative humidity increases such as in microgravity and higher radiation environment.

- $\quad$ Male's brain has more white matter and female's brain with more grey matter (Costendi, 2013) and it was discovered 
that there are $25 \%$ loss of gray matter in certain areas of schizophrenia brain (Gluck, 2019). In other words, persons with schizophrenia have reductions in gray matter in these brain regions (Kara Rogers, 2020). Reduction in gray matter in turn reduce the neurons density; and since the volume of the brain increases with increase in temperature or relative humidity while density decreases. It can be agreed upon that increase in temperature and relative humidity can decrease the level of gray matter that reduces the density of the synapses or connections between the neurons; since the male has less gray matter than the female, and male gray matter and neurons density further reduces with increase in relative humidity than female gray matter. It is also expected that the males with schizophrenia cases should be more than the females as the relative humidity increases due to reduction in the male's brain gray matter, as the synapses responsible for neurons communication with one another enhance cognition, memory formation and storage, and information processing. It has been discovered that the loss of neuron or synapses density due to reduction in gray matter has been more in males than females; this leads to cognition deficits, memory delay, declines in decision-making ability and generally negative schizophrenia symptoms in males than in females.

\section{CONCLUSION}

There are positive association between the number of male/female schizophrenia and increase in relative humidity while increase in temperature contributed nothing to the increase in the number of male and female schizophrenia patients. According to table 1, the number of female schizophrenia patients is more than the male schizophrenia patients, but the analysis with single and multivariate regression analysis shows that male population were more vulnerable at the risk of schizophrenia cases as relative humidity increases than female population. Factors such as biopsyhcosocial, environmental, and culture may not actually make male and female population equally vulnerable to the risk of schizophrenia or generally mental ill-health. In this study, the number of schizophrenia patients diagnosed and traced to increase in relative humidity and overlapping effect to be respectively 2041 and 3099 males and 1718 and 2019 females. This might be due to the fact that:

Male respond to treatment very slow compared with female; the recovery period of male with schizophrenia is longer than the female; and the rate of discharge of male schizophrenia patients from the hospital is lower than the discharge of the female schizophrenia patients.

The cognitive deficits and memory delay are more in male schizophrenia patients than the female, these can aggravate as relative humidity increases coupled with the risk of psychoactive substance use. female.

The protective effect of estrogen is lower in male than

Psychoactive substance consumption rate is higher in male than in female, and can also be at increasing rate of effect as relative humidity increases.

Testosterone, brain gray matter and neuron density reduction during hot weather or humidity are more in male than female schizophrenia patients.

\section{REFERENCES}

[1] Aleman A. (2014). Neurocognitive Basis of Schizophrenia: Information Processing Abnormalities and Clues for Treatment. Hindawi Publishing Corporation. Advances in Neuroscience. Volume 2014. Article ID 104920. 15 pages http:/dx.doi.org/10.1155/2014/104920

[2] Aleman A. et al. (2016). Involuntary Admissions in Italy: the Impact of Seasonality.

[3] Aleman A., Kahn R.S., Selten J. (2003). Sex Differences in the Risk of Schizophrenia. Arch Gen Psychiatry. 2003, 60:565-571.

[4] Amr, M. and Volpe F.M. (2012). Seasonal Influences on Admissions for Mood Disorders and Schizophrenia in a Teaching Psychiatric Hospital in Egypt. J. Affect. Disord. 2012, 137, 56-60.

[5] Barbato A. (1998). WHO. Nations for Mental Health: Schizophrenia and Public Health. WHO/MSA/NAM/97.6 English only Distr.: General.

[6] Cooper R. (2018). Extreme Heat and Mental Health: Protecting Patients. https://www.mdedge.com/psychiatry/article/168917/depression/extremeheat-and-mental-health-protecting-patients.

[7] Costendi M.O. (2013). Male Brain versus Female Brain: How do they Differ.

https://www.theguardian.com/science/neurophilosophy/2013/oct/06/malebrain-versus-female-brain

[8] Kara Rogers (2020): Encyclopaedia Britannica: Schizophrenia Psychology.

[9] Falkenburg J. and Tracy D.K. (2012). Sex and Schizophrenia: A Review of Gender Differences. Pages 61-69. Doi:10.1080/17522439.2012.733405

[10] Fernanda A.S., Mendonça D.R. Machado I.J., de Lima A.F., Gislaine M.F., Bortollotti R.C., Grilo G., dos Santos M.T. (2009). Correlation between Schizophrenia and Seasonality of Birth in a Tropical Region. Rev Brass Epidemiol 2009; 12(4):541-8.

[11] Gluck S. (2019). Schizophrenia Brain: Impact of Schizophrenia on the Brain. https://www.healthyplace.com/thought-disorders/schizophreniaeffects/schizophrenia-brain-impact-of-schizophrenia-on-the-brain

[12] Hafner H. (2002). Schizophrenia: Do Men and Women Suffer from the Same Diseases. Rev Psig Clin, 29 (6): 267-292: 2002.

[13] Jaiyeola P.O, Abdullahi A., Esu U.S. (2020). Impact of Climate Change on Psychoactive Substance Use Patients with Sex Stratifications: Variability of Temperature and Relative Humidity Effects. International Journal of Trend in Research and Development, Volume 7(1), ISSN: 2394-9333 www.ijtrd.com IJTRD

[14] Lee S., Lee H., Myung W., Kim E., Kim H. (2017). Mental diseases-related Emergence Admission Attribute to Hot Temperatures. The science of the total environment. 616-617. Doi: 10.1016/j.scitotenv.2017.10.260.

[15] Li R., Ma X., Wang G., Yang J., Wang C. (2016). Why Sex Differences in Schizophrenia? J Transl Neurosci Beijing 1(1):37-42.

[16] Novick D., Montgomery W., Treuer T., Moneta M.V., Haro J.M. (2016). Sex Differences in the Course of Schizophrenia Across Diverse Regions of the World. Neuropsychiatric Diseases and Treatment 2016.12.

[17] Ochoa S., Usall J., Cobo J., Labad X., Kulkarni J. (2012). Gender Differences in Schizophrenia and First-Episode Psychosis: A Comphensive Literature Review. Hindawi Publishing Corporation Schizophrenia Research and Treatment Volume 2012, Article ID 916198, 9 pages doi:10.1155/2012/916198.

[18] Schmeltz M.T. and Gamble J.L (2017). Risk Characteristics of Hospitalization for Mental Illness and / or Behavioral Disorders with connection to Heat Related illness. PLoS ONE 12(10): ee0186509. Doi:10.1371/journal.pone.0186509

[19] Shiloh R., Munitz H., Portuguese S., Gross-Isseroff R. et al. (2005). Corneal Temperature in Schizophrenia Patients. International Journal of $\begin{array}{llll}\text { Neuropsychopharmacology } & \text { (2005b), } & 8, & 537-547 .\end{array}$ doi:10.101'7/Si461145705005377.

[20] Shiloh R., Shapira A., Potchter O., Hermesh H., Popper M., Weizman A. (2005a). Effects of Climate on Admission Rates of Schizophrenia Patients to Psychiatric Hospitals. Eur Psychiatry. 20(1):61-64. Doi:10.1016/j.eurpsy.2004.09.020.

[21] Shiloh R., Weizman A., Stryjer R., Kahan N., Waitman D. (2009). Altered Thermoregulation in Ambulatory Schizophrenia Patients: A Naturalistic Study. World J Biol Psychiatry 2009; 10: 16370. Doi: $10.1080 / 15622970701413833$. 
[22] Xinzhu Zhang, Jian Yang, Yuhong Li1, Xin Ma1, and Rena Li1 (2017): Sex chromosome abnormalities and psychiatric diseases Oncotarget. 2017; 8:3969-3979. https://doi.org/10.18632/oncotarget.13962 Received: October 12, 2016 Accepted: December 07, 2016 Published: December 15, 2016.

[23] Zhao D., Zhang X., Xu Z., Cheng J., Xie M., Zhang H., Wang S., Li K., Yang H., Wen L., Wang X., Su H. (2016). Impact of Short-Term Temperature Variability on Emergency Hospital Admissions for Schizophrenia Stratified by Season of Birth. International Journal of Biometeorology volume 61, pages589-599.

\section{AUTHORS}

First Author - Olatunji Paul Jaiyeola, National Centre for Remote Sensing (NCRS), Jos, Nigeria

Second Author - Abdullahi Ayegba, Engineering and Space System (ESS) Department (NASRDA), Abuja, Nigeria

Third Author - Funmilola A. Oluwafemi, Engineering and Space System (ESS) Department (NASRDA), Abuja, Nigeria Fourth Author - Irene Benibo, National Centre for Remote Sensing (NCRS), Jos, Nigeria 